\title{
Non-invasive monitoring of hypoxia-inducible factor activation by optical imaging during antiangiogenic treatment in a xenograft model of ovarian carcinoma
}

\author{
B. MARTINEZ-POVEDA ${ }^{1}$, V. GOMEZ ${ }^{1}$, M.ALCAIDE-GERMAN ${ }^{1}$, S. PERRUCA ${ }^{1}$, S. VAZQUEZ ${ }^{1}$, \\ L.E. ALBA ${ }^{2}$, O.CASANOVAS ${ }^{3}$, M.L. GARCIA-BERMEJO ${ }^{4}$, L. PESO ${ }^{1}$ and B. JIMENEZ ${ }^{1}$ \\ ${ }^{1}$ Departamento de Bioquímica, Instituto de Investigaciones Biomédicas (CSIC-UAM), 28029 Madrid; \\ ${ }^{2}$ Medical Oncology Service, Virgen de Victoria University Hospital, 29010 Málaga; \\ ${ }^{3}$ Translational Research Laboratory, Catalan Institute of Oncology, IDIBELL, 08907 L'Hospitalet de Llobregat; \\ ${ }^{4}$ Department of Pathology, Hospital Ramón y Cajal, 28034 Madrid, Spain
}

Received March 24, 2011; Accepted May 16, 2011

DOI: $10.3892 /$ ijo.2011.1074

\begin{abstract}
Targeting the hypoxia response pathway and angiogenesis are two promising therapeutic strategies for cancer treatment. Their use as single strategies has important limitations. Thus, development of combined regimens has become an important step toward improving therapeutic efficacy. Also, non-invasive monitoring of the response to targeted biological therapies, as well as determination of the optimal schedule for combination regimens has become an active field of research over the last five years, with relevance for both preclinical and clinical settings. Here, we used an optical imaging method to non-invasively monitor the functional changes in HIF activity in response to antiangiogenic treatment in a xenograft model of human ovarian carcinoma. A bioluminescent reporter construct containing nine copies of the hypoxia response element upstream of the luciferase gene (9xHRE-luciferase) was characterized in vitro in a panel of tumor cell lines and in vivo in a subcutaneous xenograft model of ovarian carcinoma by means of optical imaging. We showed that in OVCAR-3 subcutaneous xenografts, the most abrupt change in the HIF functional reporter occurs before the onset of massive tumor growth. However, this system failed to detect hypoxia induced upon antiangiogenic treatment due to the compensating effects of increased hypoxia and decreased tumor cell viability caused by imbalanced neovascularization vs. tumor expansion. Therefore, the readout based on HIF functional reporter could be conditioned by the dynamics of tumor growth and angiogenesis, which is highly variable depending on the tumor type, tumor model and stage of progression.
\end{abstract}

Correspondence to: $\mathrm{Dr}$ Benilde Jimenez, Departamento de Bioquímica, Instituto de Investigaciones Biomédicas (CSIC-UAM), Arturo Duperier 4, 28029 Madrid, Spain

E-mail: bjimenez@iib.uam.es

Key words: hypoxia, antiangiogenic treatment, xenograft, ovarian carcinoma, optical imaging

\section{Introduction}

Oxygen availability is a fundamental restriction to the unlimited proliferative capacity characteristic of tumor cells. Thus, hypoxia is a common feature of most solid tumors. To surpass this barrier, tumor cells must evolve to acquire angiogenic potential, which endows them with the capacity to grow locally leading to tumor mass expansion. Therefore, suboptimal oxygen concentration (hypoxia) is one of the most relevant triggers of tumor angiogenesis and consequently the growth of tumors can be viewed as driven by cycles of hypoxia and angiogenesis. Taking these biological restrictions into account, halting angiogenesis has emerged as a general principle in which new antitumor therapies could be based.

Over the last 30 years knowledge of the molecular and cellular basis of tumor angiogenesis has led to the development of a large number of antiangiogenic strategies, some of which are at present in clinical use $(1,2)$. However, the biological output of antiangiogenic therapy has not been fully deciphered yet and may be variable depending on the tumor type, progression of the disease and antiangiogenic strategy. It is widely accepted that the dynamics of antiangiogenic drug response are initiated by an early phase of functional normalization of the tumor vasculature (early or normalization phase) characterized by an increased pericyte coverage and a decreased basal membrane thickness and vessel diameter; which results in improved tumor oxygenation (3). This functional normalization phase is followed by vessel regression caused by selective induction of apoptosis on tumor endothelial cells (late or regression phase); which results in impaired tumor perfusion and induction of necrotic tumor cell death; leading to the desired antitumor effect. Yet sustained hypoxia gives raise to one of the main modes of resistance to antiangiogenic therapy, as new inducers of angiogenesis are produced by tumor cells under the selective pressure of tumor hypoxia generated by chronic antiangiogenic treatment (4).

Monitoring of the response to antiangiogenic drugs based on diverse functional, cellular and molecular parameters is of critical importance both at preclinical and clinical settings in 
order to evaluate the benefits and limitations of antiangiogenic therapy for cancer treatment (5). Generation of tumor hypoxia by antiangiogenic treatment has been anticipated as a relevant biological output. Changes in oxygen tension are sensed by a family of HIF-prolyl hydroxylases (6-8) which, together with the product of the tumor suppressor gene VHL (9), control the stability of the $\alpha$ subunit of the hypoxia inducible factors HIF (HIF1, HIF2/EPAS and HIF3). Under hypoxia the HIF $\alpha$ subunits escape degradation, accumulate and bind to the constitutive HIF $\beta$ subunit (10). The $\alpha / \beta$ heterodimer constitutes the active HIF factor that is responsible for most of the genetic reprogramming induced to cope with fluctuations in oxygen tension deviated from the optimum. To which extent antiangiogenic treatment induces tumor hypoxia is still not properly described and could be variable depending on the antiangiogenic drug, tumor type or experimental model.

In order to address this point we used an optical imaging method to non-invasively monitor functional changes in HIF activity in response to antiangiogenic drugs in a xenograft model of human ovarian carcinoma. A bioluminescent reporter construct containing nine copies of the hypoxia response element upstream of the luciferase gene (9xHRE-luciferase) was characterized in vitro in a panel of tumor cell lines and in vivo in a subcutaneous xenograft model of ovarian carcinoma. Subsequently, the 9xHRE-luciferase reporter was used to monitor the effect of antiangiogenic treatment on HIF activity using optical imaging. Variations on the functional activity of HIF were next correlated with relevant biological responses such as angiogenesis, hypoxia and tumor cell viability monitored ex vivo by standard methods. Our results show that monitoring of the 9xHRE-luciferase reporter by optical imaging in a subcutaneous xenograft model of ovarian carcinoma allowed us to detect hypoxic areas due to an imbalance between tumor growth and new capillary formation. However, this system has limitations to detect changes in HIF activity induced by antiangiogenic treatment due to the opposing effects on the HIF reporter of simultaneous induction of hypoxia and decrease in cell viability caused by tumor vessel regression.

\section{Materials and methods}

Cell lines and reagents. Cervix (HeLa), prostate (PC3), pancreatic (BxPC3) and ovarian (OVCAR-3, SKOV-3) human carcinoma cell lines were purchased from the American Type Culture Collection (ATCC, Rockville, MD). UCD-Mel-N was derived from a patient with multiple primary melanoma tumors and generously provided by Dr E. Medrano (11). pVHLdeficient 786-O cell clones stably expressing wild-type $V H L$ (786-O-WT10) or empty plasmid (786-O-PCR3) were kindly provided by Dr W. Kaelin (Dana-Farber Institute, Boston, MA). HeLa cells were cultured in DMEM and the other cell lines were maintained in RPMI-1640. In all cases, culture media were supplemented with $100 \mathrm{U} / \mathrm{ml}$ penicillin and $100 \mu \mathrm{g} / \mathrm{ml}$ streptomycin, $2 \mathrm{mM}$ glutamine and $10 \%$ fetal bovine serum. Cells were incubated at $37^{\circ} \mathrm{C}$ in a well-humidified incubator with $5 \% \mathrm{CO}_{2}$ and $95 \%$ air.

Generation of stably transfected OVCAR-3-9xHRE-luc cells. OVCAR-3-9xHRE-luc cells expressing the luciferase gene under the control of nine tamdem copies of the hypoxia response element (9xHRE) were generated by co-transfeccion of 9xHRE-luc plasmid (12) and a hygromycin resistance plasmid at a 5:1 ratio using Lipofectamine 2000 (Invitrogen, Carlsbad, CA). After selection with $50 \mu \mathrm{g} / \mathrm{ml}$ hygromycin in culture medium, antibiotic-resistant cells were selected and established as a stable pool which was tested in vitro and used in the in vivo studies.

HIF reporter in vitro assay and detection of HIF stabilization by Western blot analysis. Cells were transfected by lipofection (Lipofectamine 2000; Invitrogen) with the HIF-responsive firefly luciferase reporter (12) and a Renilla luciferase expression plasmid at a 5:1 ratio. After transfection cells were split into 24-well plates and 20-24 h after plating, cells were exposed to hypoxia (1\% oxygen, Whitley H35 Hypoxystation; Don Whitley Scientific, UK), 1 mM DMOG (Biomol International, Plymouth Meeting, PA) or left untreated for 6 additional hours. Then, cells were harvested and firefly and Renilla luciferase activities were determined using a Dual Luciferase System (Promega, Madison, WI). Firefly luciferase activity was normalized based on the Renilla luciferase activity.

The level of HIF1- $\alpha$, HIF2- $\alpha$ and tubulin protein expression was determined by Western blotting. Anti-HIF1- $\alpha$ (Ab2185), anti-HIF2- $\alpha$ (Ab199) and anti-tubulin were purchased from Transduction Laboratories, from Novus Biologicals (Littleton, CO), and from Sigma (St. Louis, MO), respectively. Immunolabeling was detected by enhanced chemiluminescence (Amersham Pharmacia Biotechnology, Piscataway, NJ) and visualized with a digital luminescent image analyzer (Fujifilm LAS-1000 CH).

Subcutaneous xenografts and bioluminiscence imaging. OVCAR-3-9xHRE-luc cells $\left(4 \times 10^{6}\right)$ were resuspended in $100 \mu \mathrm{l}$ PBS and subcutaneously injected into the dorsal flank of 6- to 8-week-old nude mice (BALB/c nu/nu, Harlan). Monitoring of tumor-bearing mice was initiated 2 days after implantation. Tumor volume was measured every 2-3 days using a caliper and calculated using the equation: $\mathrm{D} \times(\mathrm{d})^{2} / 2$, where $\mathrm{D}$ and $\mathrm{d}$ are respectively the major and minor diameters. Animals were maintained under specific pathogen-free conditions and treated according to the protocol approved by the Universidad Autónoma de Madrid Animal Care Committee.

For the in vivo studies using antiangiogenic drugs, experimental groups $(n=5)$ were randomly established and treatments were initiated 11 days after tumor cell inoculation. Bevacizumab (Avastin ${ }^{\circledR}$, Roche) was obtained from the Pharmacy Service of Virgen de la Victoria University Hospital. It was i.p. injected every 2-3 days in a dose of $5 \mathrm{mg} / \mathrm{kg}$. ABT-510 (Abbott Laboratories, Abbott Park, IL) was injected i.p. daily in a dose of $100 \mathrm{mg} / \mathrm{kg}$. The control group was i.p. injected with an equal volume of PBS. Bioluminiscence imaging was performed as described below every 2-3 days before and during the treatment.

Twenty-five days after tumor inoculation, mice were euthanized, tumors were removed, embedded in OCT and frozen in liquid nitrogen for immunofluorescence studies.

For the in vivo bioluminiscence imaging experiments, the tumor-bearing mice were i.p. injected with $10 \mu \mathrm{l} / \mathrm{g}$ of D-luciferin solution (10 mg/ml in PBS, Promega), and subjected to imaging using an IVIS-Lumina in vivo imaging system and Living Image software (Xenogen-Calliper, San Francisco, 
CA). Bioluminescent images were acquired after the luciferin injection during $30 \mathrm{~min}$ and the maximum value of the image sequence was used for each animal.

Where indicated, animals were exposed to an $8 \% \mathrm{O}_{2}$ atmosphere for 14-16 h (Whitley H35 Hypoxystation). In these experiments, bioluminiscence values were measured just before and after exposure to hypoxia.

In vivo Matrigel plug assay. BALB/c nu/nu mice were injected s.c. with $300 \mu \mathrm{l}$ of Matrigel containing basic fibroblast growth factor (bFGF; $0.5 \mu \mathrm{g} / \mathrm{ml}$ ) alone (control) or in combination with 1-10 $\mu \mathrm{M}$ ABT-510 (ABT group). Mice were sacrificed 10 days after injection. Plugs were then removed, embedded in OCT and frozen in liquid nitrogen for immunofluorescence studies.

CD31 detection in Matrigel plugs and tumors. For immunodetection of CD31, $10 \mu \mathrm{m}$ sections were fixed in acetone, blocked with $3 \%$ BSA in PBS and incubated at $4^{\circ} \mathrm{C}$ overnight with antiCD31 antibody (BD Biosciences Pharmingen, San Deigo, CA). The anti-mouse Alexa-546 secondary antibody (Invitrogen) was used to detect the CD31 signal and DAPI to stain cell nuclei. Quantification of the CD31-positive area was performed using the NIH ImageJ analysis software. Mean values of at least 10 fields of 3 sections were calculated for each sample.

Evaluation of tumor hypoxia and quantification of tumor necrosis. To evaluate the presence of hypoxic areas in tumors we used the nitroimidazole derivative pimonidazole (Hypoxyprobe ${ }^{\mathrm{TM}}$, HPI, Inc.). Pimonidazole hydrochloride $(60 \mathrm{mg} / \mathrm{kg})$ was injected i.v. into tumor-bearing mice and after $1 \mathrm{~h}$ animals were sacrificed. Excised tumors were fixed in $4 \%$ paraformaldehyde, embedded in OCT and frozen in liquid nitrogen. Sections of $10 \mu \mathrm{m}$ were prepared and immunodetection of pimonidazole was performed using anti-pimonidazole antibody (HPI, Inc.). Images were acquired using a Nikon TS100 (Nikon, Inc., Melville, NY) inverted phase contrast microscope with a DS-5M-L1 (Nikon) photomicrographic camera system. Total hypoxic area in tumor sections was determined by scoring diaminobenzidine (DAB)-positive pixels in x100 magnification images using the NIH ImageJ analysis software. Two tumor sections were analyzed for each experimental condition and the mean value \pm SD was determined

Tumor necrosis was evaluated by standard hematoxylin and eosin (H\&E) staining. Brieftly, frozen sections were thawed, fixed in cold acetone, stained using Mayer's hematoxylin for $1 \mathrm{~min}$ and counterstained with eosin. Quantification was performed as indicated for pimonidazole staining. Four tumors were analyzed for each experimental condition and the mean value $\pm \mathrm{SD}$ was determined

\section{Results}

In vitro functional evaluation of 9xHRE-luciferase reporter in a panel of tumor cell lines. Generation of hypoxic areas in tumors can be quantified indirectly by using reporter vectors in which the reporter gene is controlled by a tandem of hypoxia-response elements (HREs). We used the 9xHREluciferase (9xHRE-luc) reporter vector that harbors nine copies of the HRE derived from the VEGFA gene upstream of a minimal rat prolactin promoter driving the expression of the firefly luciferase gene (12). Similar vectors, containing a variable number of HREs and diverse reporter genes (luciferase or EGFP) have been developed and used as functional reporters for optical imaging over the last 5 years (13-19). Hypoxic conditions lead to hypoxia inducible factor (HIF) stabilization and subsequent induction of luciferase reporter gene expression through binding of HIF to HREs in the reporter and activation of transcription driven by HIF.

We first evaluated the suitability of 9xHRE-luc as a bona fide hypoxia inducible reporter in a panel of human tumor cell lines transiently transfected with 9xHRE-luc; including melanoma (UCD-Mel-N), cervix (HeLa), prostate (PC3), renal (786-O-PRC3, 786-O-WT10), pancreatic (BxPC3) and ovarian (OVCAR-3, SKOV-3) carcinomas. Reliability of our reporter system was demonstrated by lack of basal activity in 786-O-WT10 cells as compared to 786-O-PRC3 cells (Fig. 1A, left graph). 786-O-PRC3 cells are derived from a clear cell renal carcinoma lacking functional VHL protein. pVHL is the substrate recognition subunit of an E3-ubiquitin ligase complex that targets HIF for proteasomal degradation under normoxia. Thus, 786-O-PRC3 cells show a high basal activity of HIF that is abolished upon forced expression of a normal VHL gene as is the case of the 786-O-WT10 clone. Analysis of these results (Fig. 1A, left graph) revealed a subset of tumor cell lines in which the basal level of the reporter activity was very low (HeLa and OVCAR-3) as compared to others that displayed significant basal levels of HIF-mediated transcription (PC3, SKOV-3, BxPC3, UCD-Mel-N). The increased basal activity observed in PC3 cells can be explained by the amplification of the HIF locus described in this cell type (20). Induction of 9xHRE-luc reporter by hypoxia (Fig. 1A, middle graph) and the hypoxia mimetic DMOG (Fig. 1A, right graph) ranged from 10- to 100-fold and the stabilization of HIF1- $\alpha$ or HIF2- $\alpha$ under hypoxia was confirmed by Western blot analysis (Fig. 1B), with the induction of HIF- $\alpha$ being more evident in most cell lines.

Based on these results, the ovarian carcinoma cell line OVCAR-3-9xHRE-luc was selected for subsequent in vivo studies due to its low basal HIF activity and large inducibility of the reporter upon hypoxia.

In vivo functional evaluation of 9xHRE-luciferase reporter by optical imaging in subcutaneous xenografts of ovarian carcinoma. 9xHRE-luc reporter was next evaluated in vivo using bioluminescence optical imaging in a subcutaneous xenograft model of ovarian carcinoma. To this end we generated the OVCAR-3-9xHRE-luc cell line by stable transfection of OVCAR-3 cells with the 9xHRE-luc construct. This cell line was injected s.c. in nude mice and the bioluminiscent signal (Total Flux, photons/sec) was monitored, on a daily basis, after the administration of D-luciferin. A sequence of optical images and the corresponding data of Total Flux vs. time after implantation are shown in Fig. 2A. The dynamics of 9xHREluc reporter activity in growing tumors displayed three distinct phases. We first observed a gradual decrease in Total Flux corresponding to the initial phase of establishment of the tumor (from the day of s.c. injection up to day 7-10) during which many tumor cells died ('9xHRE-luc reporter establishment phase', data not shown). This was followed by a large increase in Total Flux (Fig. 2B left, Total Flux vs. tumor size logarithmic scale) in the absence of detectable tumor growth 
A

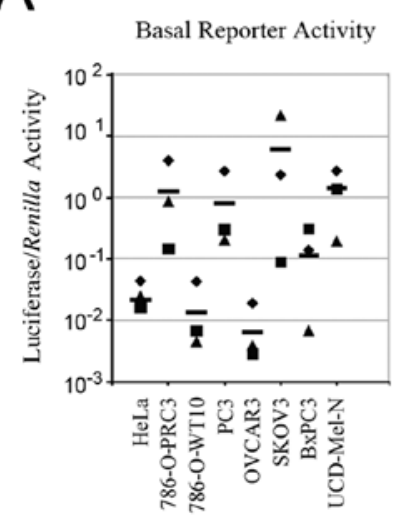

B
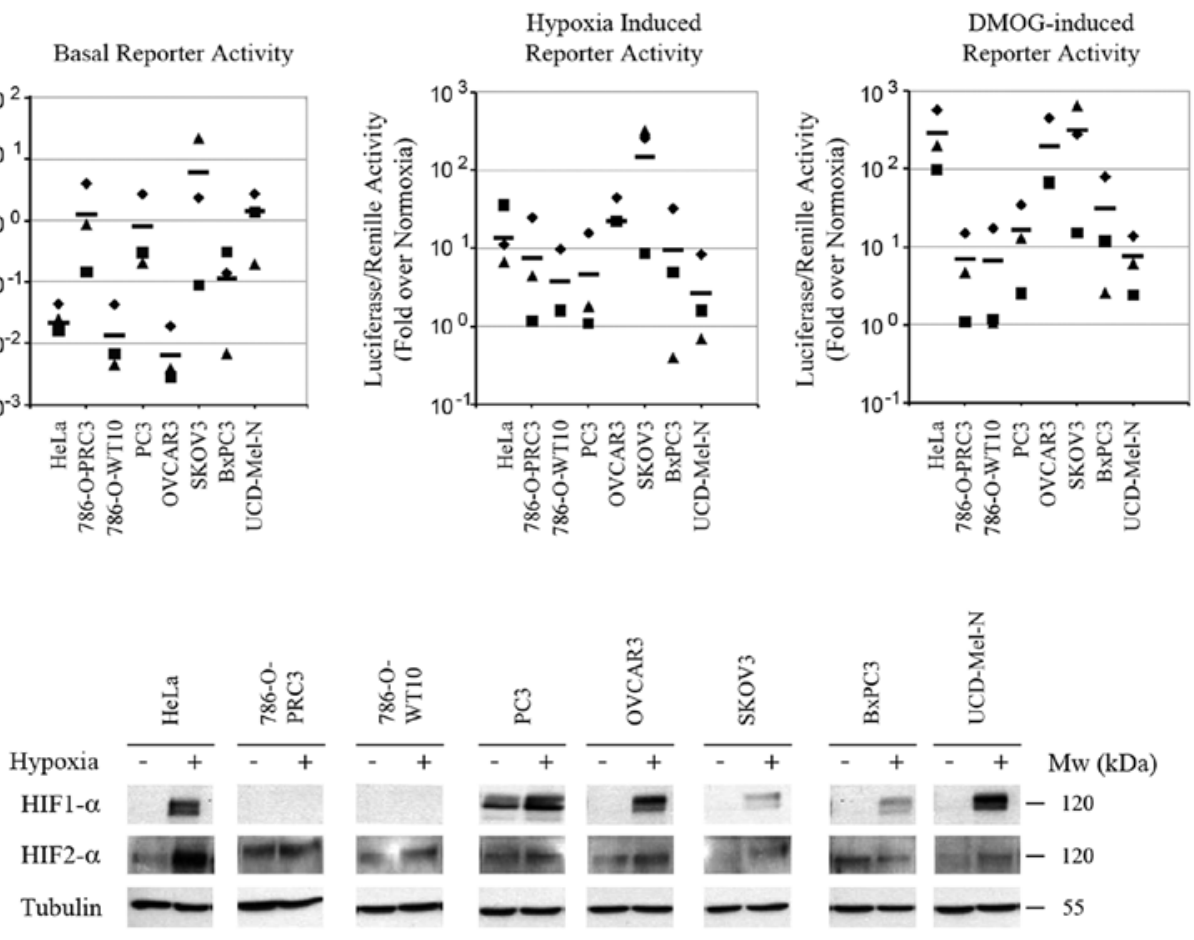
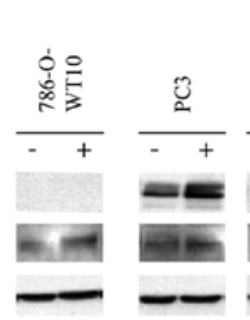
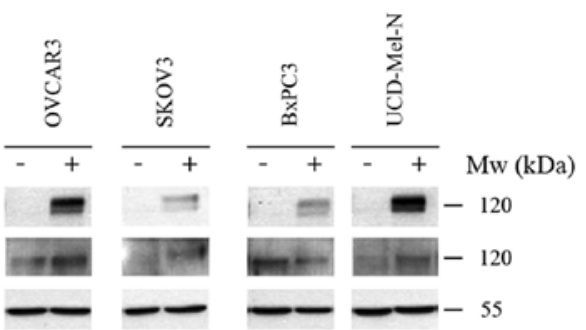

Figure 1. In vitro characterization of the 9xHRE-luciferase reporter in a panel of tumor cell lines. (A) The activity of the 9xHRE-luciferase reporter was measured in a panel of tumor cell lines including melanoma (UCD-Mel-N), cervix (HeLa), prostate (PC3), renal (786-O-PRC3, 786-O-WT10), pancreatic (BxPC3) and ovarian (OVCAR-3, SKOV-3) carcinoma cell lines, transiently transfected with the 9xHRE-luc reporter contruct. Basal activity (left graph), fold induction by hypoxia (middle graph) and fold induction by DMOG (right graph) are shown. Graphs represent values obtained from three independent experiments $(\boldsymbol{\Lambda}, \mathbf{\square}, \bullet)$ and their mean (-). In the middle and right graphs the values of luciferase activity (corrected by Renilla activity) upon treatments (hypoxia/ DMOG) are represented as the fold over the the normoxic values. (B) HIF1- $\alpha$ and HIF2- $\alpha$ stabilization by hypoxia was evaluated by Western blot analysis in the panel of tumor cell lines used in (A). Tubulin was used as loading control.

('9xHRE-luc reporter exponential phase'). This initial burst of reporter activity was followed by a gradual/linear increase in Total Flux (Fig. 2B right, Total Flux vs. tumor size, linear scale) that paralleled an increase in tumor size ('9xHRE-luc reporter linear phase').

In vivo induction of 9xHRE-luc reporter by hypoxia in the tumor setting was directly demonstrated by introducing tumor bearing mice in a hypoxia work station. As shown in Fig. 2C, the Total Flux in tumors from animals exposed to an $8 \%$ oxygen atmosphere for 14-16 h (hypoxic condition) increased 16-fold relative to Total Flux in tumors from animals kept at normoxic conditions.

Together these results indicate that the system based on the 9xHRE-bioluminiscent reporter is able to monitor changes in tumor hypoxia in vivo by means of optical imaging. This system allowed us identify a specific pattern of hypoxia dynamics during the growth of subcutaneous OVCAR-3 xenografts.

Monitoring of the response to antiangiogenic treatment by optical imaging using the 9xHRE-luciferase reporter in subcutaneous xenografts of ovarian carcinoma. We next investigated the suitability of this reporter to indirectly detect variations in tumor oxygenation caused by treatment with two types of antiangiogenic strategies: i) bevacizumab, a humanized monoclonal antibody against vascular endothelial growth factor-A and ii) ABT-510, a peptide mimetic of the endogenous inhibitor of angiogenesis thrombospondin-1. Antiangiogenic treatment was initiated on day 11 after the inoculation of tumor cells in nude mice; therefore, our experimental design was intended to evaluate the effect of inhibition of angiogenesis on the '9xHRE-luc reporter linear phase'. Comparison of Total Flux vs. tumor size in the three experimental groups (control, bevacizumab and ABT-510) showed that, in spite of a similar initial 'exponential phase' (Fig. 3A, lower panels), bevacizumab induced a decrease in large tumors corresponding to the 'linear phase' (Fig. 3A, upper panels), while there was no significant effect of ABT-510 in the subcutaneous xenograft ovarian carcinoma model (Fig. 3A, top panels). Representation of variation of Total Flux during the treatment period (increase in Total Flux from day 11 to day 25) for the three experimental groups confirmed that bevacizumab, but not ABT-510, treatment significantly $(\mathrm{p}<0.05)$ reduced Total Flux (Fig. 3B).

A potential explanation for this unexpected result is that D-luciferin delivery to hypoxic regions could be compromised by the antiangiogenic effect of bevacizumab treatment. However, exposure of mice to hypoxia led to a similar induction of 9xHRE-luc reporter in tumors from control, ABT-510 and bevacizumab groups (Fig. 3C), arguing against such a possibility.

Ex vivo evaluation of antiangiogenic treatment in subcutaneous xenografts of ovarian carcinoma. We next intended to correlate variations in 9xHRE-luc reporter in response to bevacizumab 
A
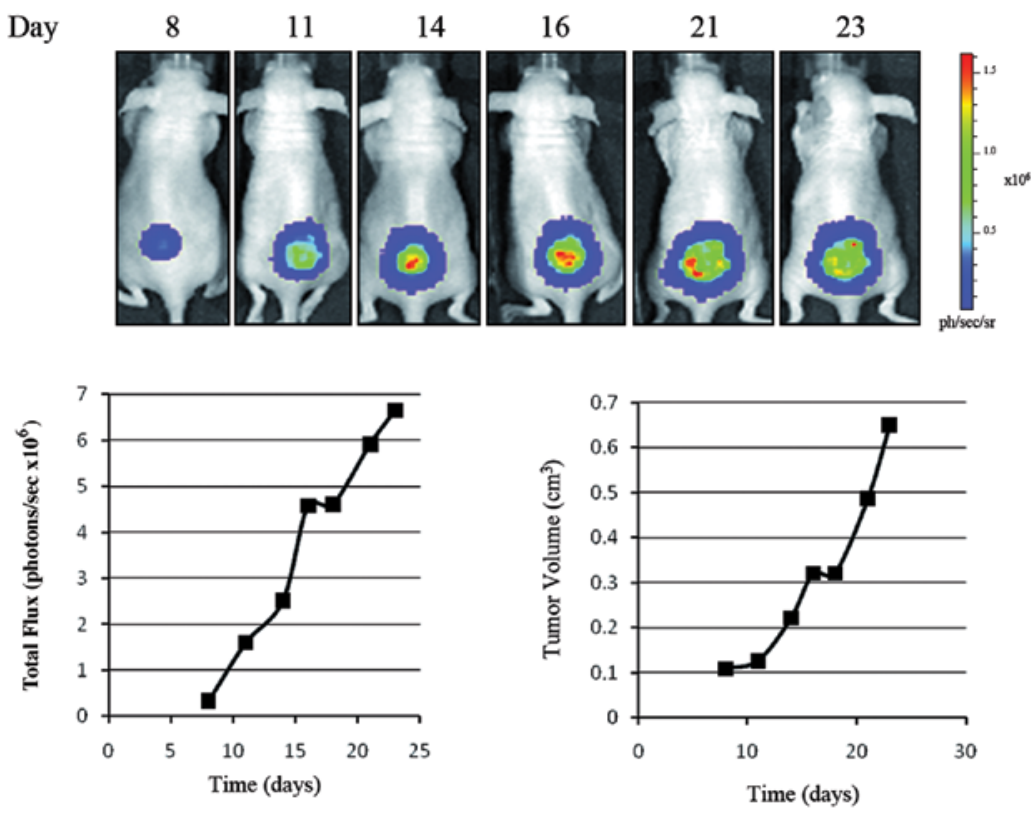

B
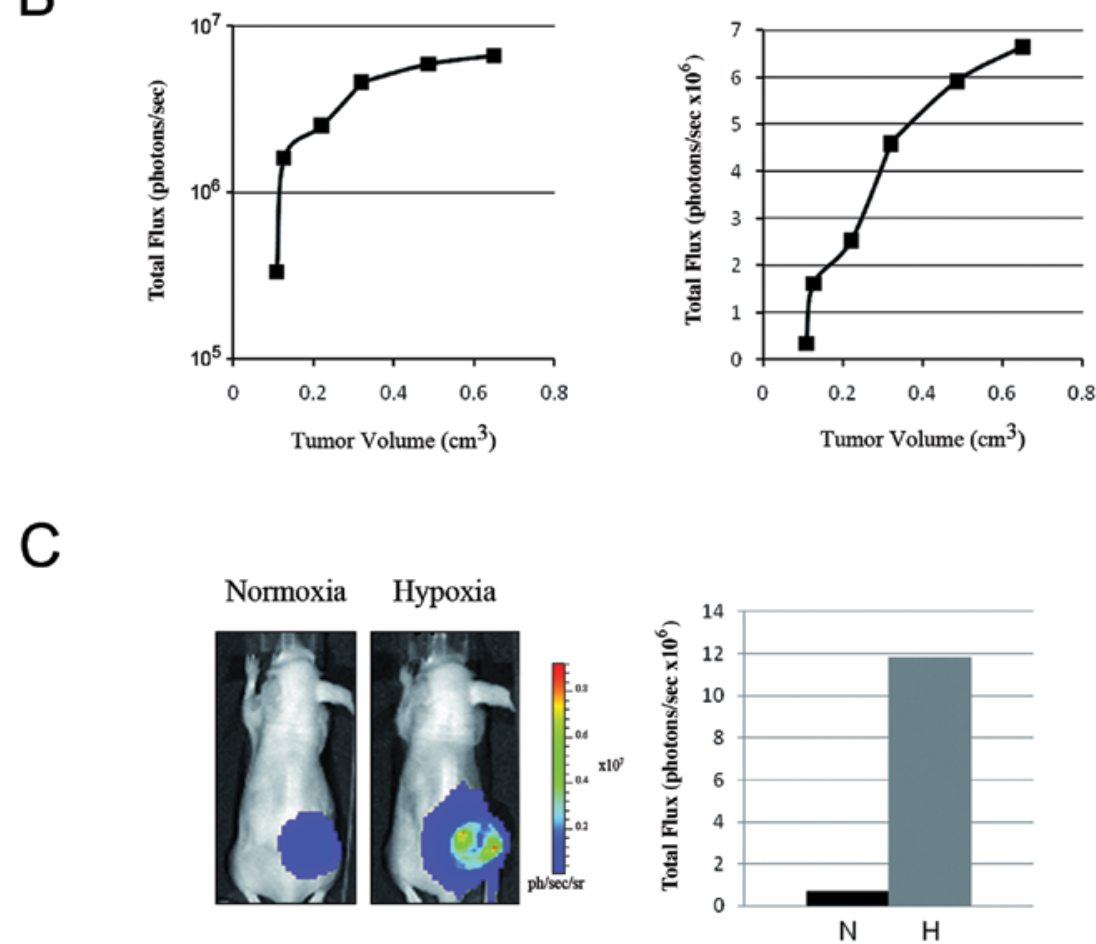

Figure 2. Functional evaluation of the 9xHRE-luciferase reporter by bioluminescence imaging during in vivo growth of subcutaneous xenografts of OVCAR-3-9xHRE-luc cells. (A) Activity of 9xHRE-luciferase reporter was monitored by bioluminescence imaging during growth of subcutaneous xenografts of OVCAR-3-9xHRE-luc cells in immunodeficient mice. Mice were imaged on day 8 and subsequently every 1-3 days until day 23 . A temporal sequence of images of a representative tumor is shown (upper panels). Total bioluminiscence photon counts (Total Flux, photons/sec) and tumor size $\left(\mathrm{cm}^{3}\right)$ were represented vs. time (days) (lower left and right graphs, respectively). (B) Total bioluminiscent photon counts (Total Flux, photons/sec) were represented vs. tumor size $\left(\mathrm{cm}^{3}\right)$ using a logaritmic scale (left) or a linear scale (right). (C) Induction of 9xHRE-luciferase reporter in subcutaneous tumors by hypoxia in vivo. Mice were kept under hypoxia for $16 \mathrm{~h}$ prior to D-luciferin injection and imaging. Representative images of tumors before (normoxia) and after hypoxia are shown (left). Total bioluminiscence photon counts (Total Flux, photons/sec) were represented for tumors in normoxia $(\mathrm{N})$ and hypoxia $(\mathrm{H})(\mathrm{right})$.

with relevant biological outputs that might be predictive of a biological response to bevacizumab; such as tumor angiogenesis, tumor hypoxia and tumor cell viability evaluated ex vivo in tumor sections by standard methods.
Bevacizumab treatment of subcutaneous tumors of OVCAR-3-9xHRE-luc cells reduced tumor microvascular density by $\sim 2$-fold after 14 days of treatment (Fig. 4 A, top images and lower left graph). The response to ABT-510 in the 
A

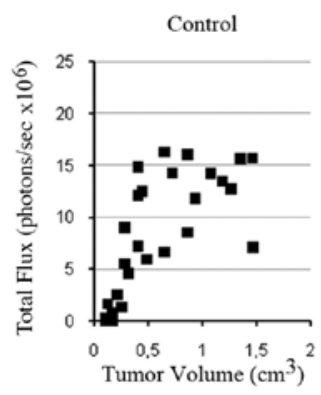

Control

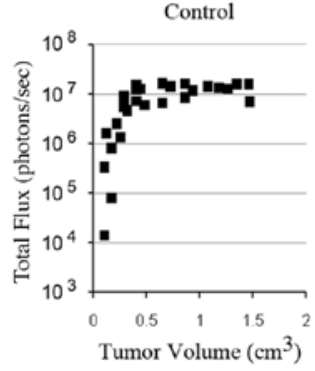

B

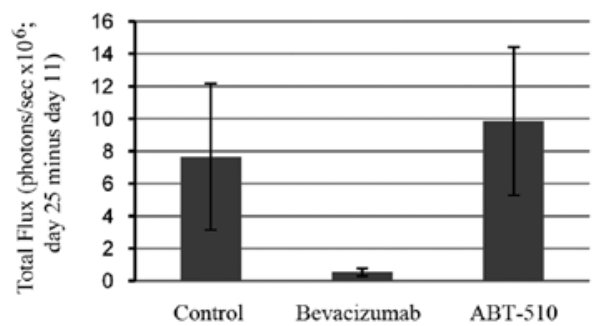

Bevacizumab

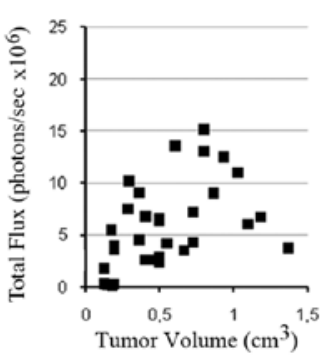

Bevacizumab

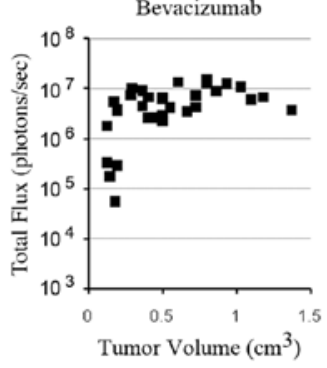

$\mathrm{ABT}-510$

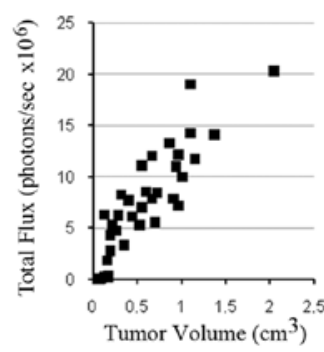

ABT-510

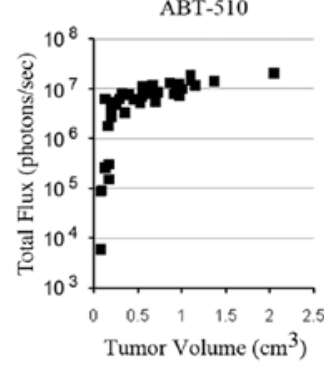

C

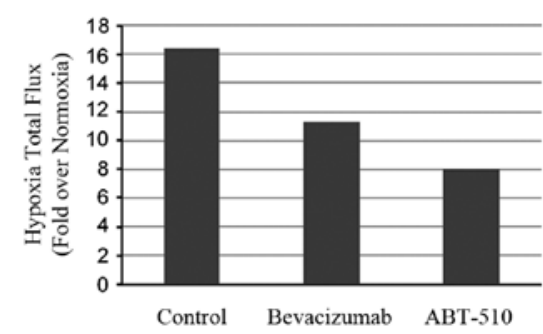

Figure 3. Real-time, non-invasive monitoring of the effect of antiangiogenic treatment on HIF1- $\alpha$ activation by bioluminescent imaging in subcutaneous xenografts of OVCAR-3-9xHRE-luc cells. (A) Total bioluminiscence photon counts (Total Flux, photons/sec) were represented vs. tumor size (cm ${ }^{3}$ ) for control (left), bevacizumab (middle) and ABT-510 (right) using a linear scale (upper graphs) or a logaritmic scale (lower graphs). (B) Mean total bioluminiscence photon counts at day 25 (end of experiment) minus day 11 (beginning of antiangiogenic treatment), were represented for each experimental group (control, bevacizumab, ABT-510). The graph represent the average values for each group and their standard deviation. (C) Total bioluminiscence photon counts (Total Flux, photons/sec) were determined in mice from each experimental group (control, bevacizumab, ABT-510) exposed to hypoxia at the end of the experiment (day 25). The graph represents bioluminiscence photon counts after 16-h exposure to hypoxia as a fold over the counts prior to exposure to hypoxia.

OVCAR-3-9xHRE-luc tumors was highly variable and there were no significant differences in the mean tumor microvascular density between control and ABT-510 groups (Fig. 4A). In contrast, ABT-510 showed a marked antiangiogenic effect in vivo in a matrigel plug assay (Fig. 4B), confirming that this reagent has the expected biological activity. Hence, these result suggested that OVCAR-3 subcutaneous xenografts were sensitive to bevacizumab, but not ABT-510, treatment.

In agreement with their effect on the vasculature of OVCAR-3-derived tumors, bevacizumab, but not ABT-510, treatment consistently reduced tumor size, while there was no relevant change in the ABT-510 experimental group compared to the control group (Fig. 4A, lower right graph). Despite of their trend, differences did not reach statistical significance due to the variability in the control group.

Next, we analyzed the effect of antiangiogenic treatment on tumor hypoxia by injection of the nitroimidazole derivative pimonidazole $1 \mathrm{~h}$ before tumor collection and subsequent detection of pimonidazole adducts by staining with a peroxidase-conjugated hypoxyprobe antibody. Growth of OVCAR-3-9xHRE-luc tumors significantly induced tumor hypoxia. Significant fields for the control, bevacizumab and ABT-510 experimental groups are shown for day 30 in Fig. 5A. Also a representative field for pimonidazole staning for the control group at day 11 was included in Fig. 5A (upper left image). We monitored possible differences in pimonidazole staining on day 30 between the control, ABT-510 and bevacizumab groups. We found no significant differences comparing the control with ABT-510 (Fig. 5A). Surprisingly, tumors from bevacizumab-treated mice were negative for pimonidazole staining (Fig. 5A). This result could be explained by compromised pimonidazole delivery due to tumor vessel regression caused by bevacizumab treatment as was recently described in a model of PC3 carcinoma (21). However, monitoring of the HIF reporter was not compromised in the bevacizumab-treated group (Fig. 3B) arguing against this possibility.

Finally, the effect of antiangiogenic treatment on tumor cell viability was estimated by $\mathrm{H} \& \mathrm{E}$ staining. Consistent with 
A
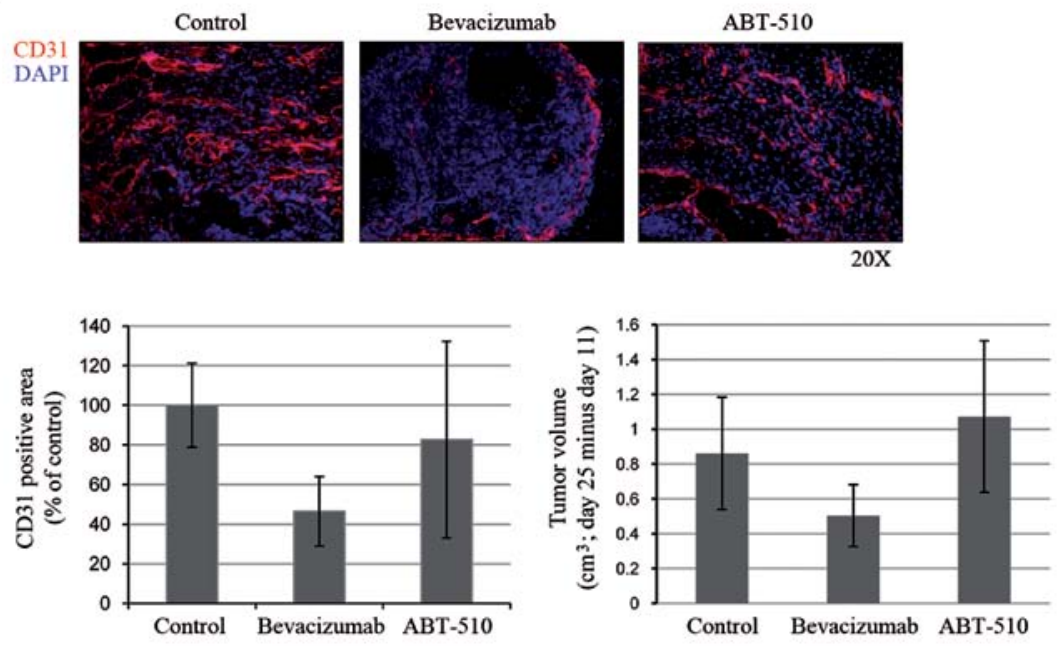

B
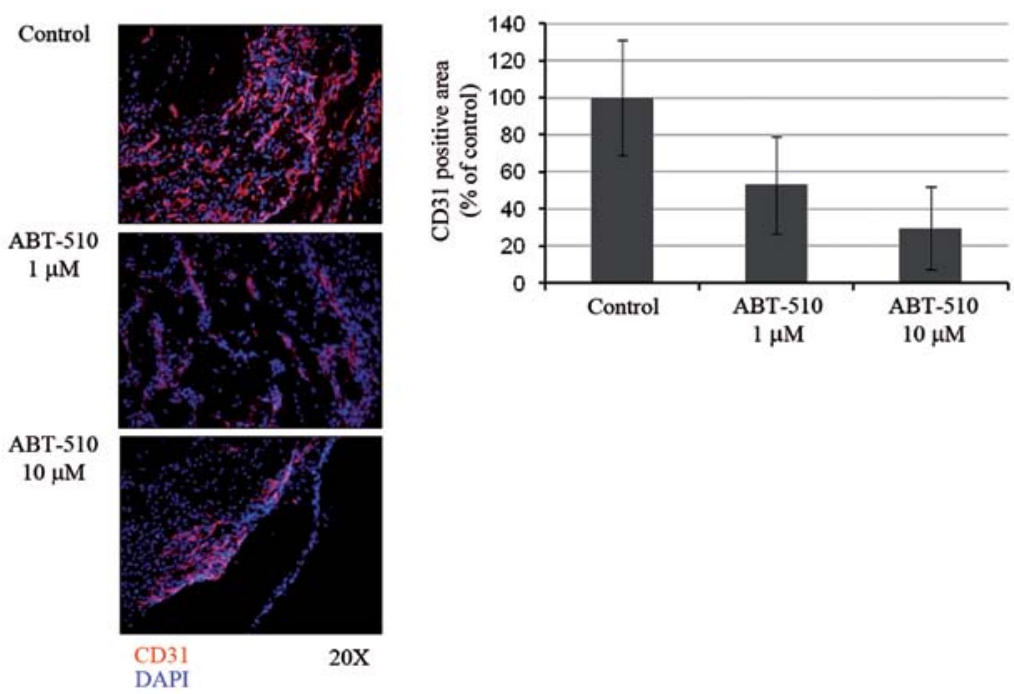

Figure 4. Quantification of the antiangiogenic effect of bevacizumab and ABT-510. (A) The antiangiogenic effect of bevacizumab and ABT-510 on subcutaneous xenografts was evaluated by CD31 immunostaining of tumor sections. DAPI staining was used to evaluate the cellular density in selected fields. Representative images of tumor sections from control, bevacizumab or ABT-510 experimental groups are shown (upper panels). Percentage of CD31 positive staining is shown relative to the control group (lower graph, left). The graph represents the average values obtained in 10 independent fields and the standard deviation. Mean tumor size at day 30 (end of experiment) minus day 11 (beginning of antiangiogenic treatment), was represented for each experimental group (control, bevacizumab, ABT-510) (lower right graph). (B) The biological activity of ABT-510 was verified using the Matrigel plug assay. Angiogenesis was evaluated by CD31 immunostaining of plug sections. DAPI staining was used to evaluate cellular density in selected fields. Representative images of plug sections from control (bFGF), bFGF plus ABT-510 at different concentrations are shown (left panels). Percentage of CD31 positive staining is shown relative to the control group (right graph). The graph represents the average values obtained in 10 independent fields and the standard deviation.

the induction of hypoxia during the growth of OVCAR-39xHRE-luc tumors, there was an increase in the percentage of necrotic tumor areas (Fig. 5B). Induction of necrosis was highly variable among different types of subcutaneous tumors in immunodeficient mice; ranging from no necrosis such as in HeLa and UCD-Mel-N tumors (data not shown), to highly represented necrosis in OVCAR-3 tumors (Fig. 5B). Treatment of OVCAR3-9xHRE-luc tumors with bevacizumab, but not ABT-510, resulted in a further 15\% increase in the necrotic area (Fig. 5C). We observed that changes induced by bevacizumab in 9xHREluc reporter activity were larger than the reduction of tumor size caused by bevacizumab treatment (Fig. 3A). This discrepancy could be explained by the decreased number of viable tumor cells in the bevacizumab treated group.

\section{Discussion}

As a result of uncontrolled proliferation, tumor cells outgrow their vascular supply territories, thus originating hypoxic areas within the tumor mass. The adaptation of tumor cells to decreased oxygen availability is mastered by hypoxia inducible factors (HIFs), which coordinate an extensive reprogramming of gene expression aimed to adjust metabolism to suboptimal oxygen concentrations and to increase oxygen 
A

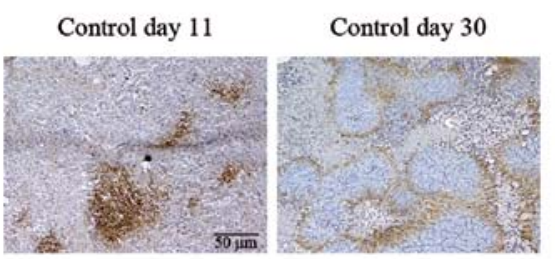

Bevacizumab day 30

ABT-510 day 30
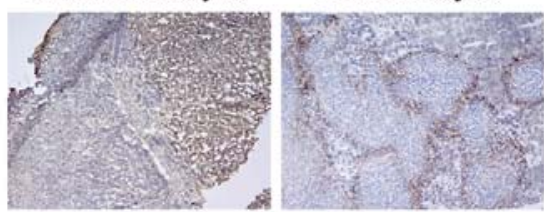

$100 \mathrm{X}$

B
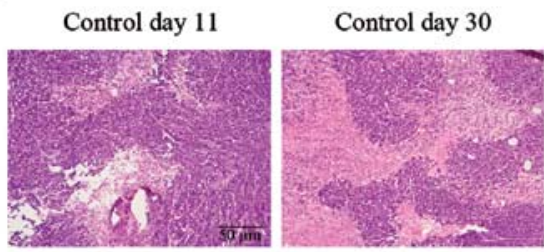

Bevacizumab day 30

ABT-510 day 30
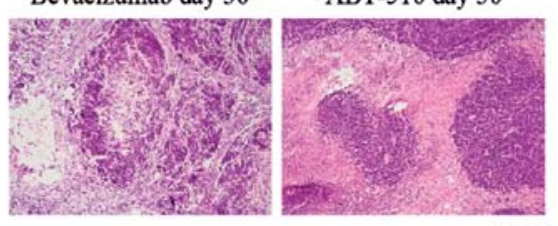

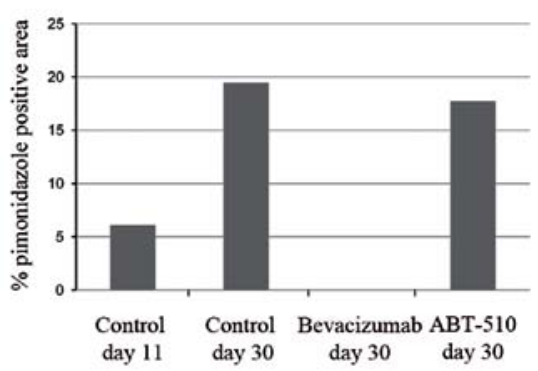

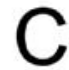

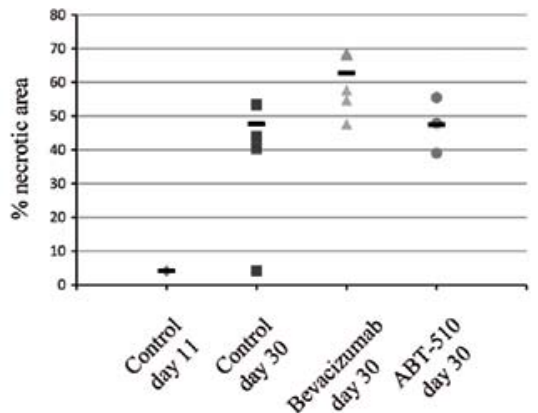

Figure 5. Quantification of the effect of antiangiogenic treatment on tumor hypoxia and necrosis. (A) Hypoxia was evaluated using pimonidazole staining. (B) Necrosis was evaluated by H\&E staining. (A, B) Representative images are shown for the control group at day 11 before initiating treatment (control day 11 ) and at day 30 for the control group (PBS treated) (control day 30) and, bevacizumab and ABT-510-treated groups. Magnification is indicated on the images. Graphs represent the percentage of hypoxic (A) and necrotic (B) area for each experimental group (control, bevacizumab and ABT-510).

delivery by inducing the formation of new blood vessels. Furthermore, in the tumor context hypoxic driven reprogramming correlates with increased metastatic potential and highly hypoxic tumors are often associated with poor prognosis and worse response to chemotherapy or radiotherapy (22).

Relevance of tumor hypoxia and HIFs in cancer progression has led to the development of HIF-targeting strategies $(17,22-24)$ and non-invasive in vivo imaging techniques to monitor intratumoral hypoxia or HIF stabilization or activation (25-27). In addition to the activation of the HIF pathway as a consequence of tumoral hypoxia, specific genetic lesions lead to the constitutive stabilization of HIF, the paradigm being the inactivation of VHL in clear cell carcinomas and pheochromocytomas (28). Moreover, the constitutive activation of HIF seems to be central to the generation and progression of some tumors (29). In this regard, we found that several tumoral cell lines displayed high basal (normoxic) HIF activity (Fig. 1A). In the case of the prostate cancer PC3 cells, this activity is expected to be a consequence of the amplification of the HIF locus described for this cell type (20). In agreement, PC3 cells display a high basal level of HIF1- $\alpha$ protein (Fig. 1B). However, we do not know the reason for the high basal HIF activity found in SKOV-3, BxPC3 and UCD-Mel-N cells. Intriguingly, in these cases, increased HIF activity is not paralleled by accumulation of HIF $\alpha$ proteins. Regardless of the molecular mechanism, our results suggest that constitutive HIF activity could be a more general feature of tumors than previously recognized, underlying the importance of this molecule as a therapeutic target.

Effectiveness of some HIF targeting strategies (23) could be improved by combination with antiangiogenic strategies, which are expected to further increase tumor hypoxia. To this end monitoring of the impact of antiangiogenic therapy on the HIF pathway is of critical importance for the evaluation of the tumor's response to the antiangiogenic regimen and to define the right schedule for the combination therapy using HIF inhibitors. Here we used an optical imaging method to noninvasively monitor functional changes in HIF activity during growth of subcutaneous xenografts of the ovarian carcinoma cell line OVCAR-3 and in response to antiangiogenic therapy.

OVCAR-3 cells cultured in vitro under normoxic conditions displayed very low basal levels of our functional bioluminiscent HIF reporter (9xHRE-luc) and high inducibility (30-fold) under hypoxic conditions. Remarkably, in vivo, HIF reporter activity increased by orders of magnitude without apparent tumor 
growth at early time-points during tumor progression (Figs. 2 and 3). This dramatic change in HIF reporter activity was followed by a linear increase in reporter activity that paralleled an increase in tumor size (Fig. 3). Our interpretation of this sharp increase of reporter activity is that it preceded the tumor angiogenic switch and, therefore, was a consequence of hypoxia caused by initial tumor growth in the absence of angiogenesis. The subsequent linear increase in the HIF reporter could be explained by a number of interpretations: i) although 9xHREluc reporter was strictly silent in OVCAR-3 cells in vitro, we cannot rule out a significant basal activity of the reporter in vivo, ii) compensation of hypoxia by neovascularization once the angiogenic switch is operating and iii) secondary peaks of HIF reporter could be masked by decreased cell viability (necrosis). The reported dynamics of functional HIF reporters monitored by optical imaging were highly variable depending on the tumor model: subcutaneous allograft (26) vs. subcutaneous orthotopic xenograft (24) or our data in subcutaneous xenografts. This variability most probably reflects differences in tumor growth rate and patterns of tumor vascularization. In our study, OVCAR-3 subcutaneous xenografts displayed significant necrotic areas, despite being highly vascularized tumors; pointing to exacerbated but defective tumor angiogenesis unable to cope with the tumor expansion rate. These characteristics could underlie the gradual increase in HIF reporter even in large tumors, where activation of the reporter by hypoxia could be compensated by increased neovascularization, followed by decreased number of viable cells due to deficient perfusion. In agreement, Fig. 5A shows that pimonidazole staining increases during tumor growth, which is compatible with the trend observed for the reporter at those time-points (Fig. 2B).

As OVCAR-3 tumors were highly vascularized, we expected a good response to antiangiogenic therapy, which was confirmed in the case of bevacizumab treatment. Unexpectedly, we detected a gradual decrease in HIF reporter activity over the duration of bevacizumab treatment. In the few publications that evaluated the effect of bevacizumab using a luminescent HIF reporter results were divergent: activation of the reporter in a lung adenocarcinoma model (15) and inhibition of the reporter in a glioblastoma model (16). A decrease in HIF reporter activity by antiangiogenic treatment could be explained by: i) a decrease in intratumoral hypoxia caused by vascular normalization; ii) an increase in intratumoral hypoxia that may cause a decrease in luciferase activity due to limited oxygen availability; iii) reduced availability of luciferase substrate as a consequence of reduced vascularization; and iv) an increase in intratumoral hypoxia compensated with a decrease in the number of viable cells. In our study we reasoned that: i) vascular normalization was an unlikely explanation, as the decrease in HIF reporter imposed by bevacizumab treatment lasted 14 days; and ii) oxygen/luciferin availability was also ruled out, as HIF reporter was activated to the same extent by external hypoxia imposed on bevacizumab, ABT-510 and control mice (Fig. 3C); and iii) the compensation on HIF reporter activity of opposing increased intratumoral hypoxia and decreased tumor size caused by induction of necrosis by bevacizumab treatment which was the favored explanation based on evaluation of biological parameters in OVCAR-3 tumors ex vivo. A further factor that could contribute to loss of reporter signal in large tumors treated with bevacizumab is the inactivation of the HIF pathway as consequence of exposure to sustained hypoxia. In fact, in vitro experiments have demonstrated the existence of a HIF-mediated negative feed-back loop (30). Moreover, HIF-mediated metabolic adaptation results in lower oxygen consumption by the cells and thus higher availability of oxygen that could lead to HIF-degradation. Regardless of the mechanism, the inactivation of HIF activity upon sustained hypoxia in vivo has been previously reported (26). Finally, consistent with unresponsiveness of OVCAR-3 tumors to an alternative antiangiogenic treatment using ABT-510, no differences in HIF reporter activity or percentage of necrotic areas were detected in the ABT-510 group compared to the control group. In contrast to our data, it has been recently demonstrated that, in an orthotopic syngeneic mouse model of ovarian cancer, ABT-510 treatment reduced tumor microvessel density and increased the percentage of mature blood vessels (31) resulting in increased efficacy of chemotherapeutic drugs when used in combination with ABT-510 (32). Lack of responsiveness to ABT-510 in our model of subcutaneous xenotransplant could be attributed to significant differences in the pattern of tumor vascularization or angiogenic balance.

In summary, our results showed that, in OVCAR-3 subcutaneous xenografts, the most abrupt change in HIF functional reporter occurs before the onset of massive tumor growth. Subsequent peaks of HIF reporter activation may be masked by decreased tumor cell viability induced by tumor hypoxia caused by tumor growth or forced by antiangiogenic treatment. Although a number of articles have been published over the last five years addressing the functional monitoring of HIF stabilization or HIF activation by optical imaging $(19,24,26,27,33,34)$; very few studies have investigated the usefulness of these functional reporters to monitor changes in HIF stabilization induced by antiangiogenic treatment. Further analysis in a variety of tumor models and using diverse antiangiogenic strategies is required to expand our view of the usefulness and possible limitations of functional HIF reporters to monitor changes in HIF induced by antiangiogenic treatment.

\section{Acknowledgements}

This study was supported by grants from the Ministerio de Ciencia y Tecnología/Ministerio de Ciencia e Innovación (SAF2008-03147 to LdP and SAF2010-19256 to BJ), Comunidad Autónoma de Madrid (S-SAL-0311_2006) and the 7th Research Framework Programme of the European Union (METOXIA, project ref. HEALTH-F2-2009-222741). B.M.P. and V.G. have been supported by a grant from the Comunidad Autónoma de Madrid (S-SAL-0311_2006).

\section{References}

1. Ferrara $\mathrm{N}$ and Kerbel RS: Angiogenesis as a therapeutic target. Nature 438: 967-974, 2005.

2. Kerbel RS: Tumor angiogenesis. N Engl J Med 358: 2039-2049, 2008.

3. Jain RK: Normalization of tumor vasculature: an emerging concept in antiangiogenic therapy. Science 307: 58-62, 2005.

4. Bergers $G$ and Hanahan D: Modes of resistance to anti-angiogenic therapy. Nat Rev Cancer 8: 592-603, 2008.

5. Jain RK, Duda DG, Willett CG, et al: Biomarkers of response and resistance to antiangiogenic therapy. Nat Rev Clin Oncol 6: 327-338, 2009. 
6. Bruick RK and McKnight SL: A conserved family of prolyl4-hydroxylases that modify HIF. Science 294: 1337-1340, 2001.

7. Epstein AC, Gleadle JM, McNeill LA, et al: C. elegans EGL-9 and mammalian homologs define a family of dioxygenases that regulate HIF by prolyl hydroxylation. Cell 107: 43-54, 2001.

8. Ivan M, Haberberger T, Gervasi DC, et al: Biochemical purification and pharmacological inhibition of a mammalian prolyl hydroxylase acting on hypoxia-inducible factor. Proc Natl Acad Sci USA 99: 13459-13464, 2002.

9. Maxwell PH, Wiesener MS, Chang GW, et al: The tumour suppressor protein VHL targets hypoxia-inducible factors for oxygen-dependent proteolysis. Nature 399: 271-275, 1999.

10. Coleman ML and Ratcliffe PJ: Oxygen sensing and hypoxiainduced responses. Essays Biochem 43: 1-15, 2007.

11. Reed JA, Bales E, Xu W, Okan NA, Bandyopadhyay D and Medrano EE: Cytoplasmic localization of the oncogenic protein Ski in human cutaneous melanomas in vivo: functional implications for transforming growth factor beta signaling. Cancer Res 61: 8074-8078, 2001.

12. Aragones J, Jones DR, Martin S, et al: Evidence for the involvement of diacylglycerol kinase in the activation of hypoxia-inducible transcription factor 1 by low oxygen tension. J Biol Chem 276: 10548-10555, 2001.

13. Cecic I, Chan DA, Sutphin PD, et al: Oxygen sensitivity of reporter genes: implications for preclinical imaging of tumor hypoxia. Mol Imaging 6: 219-228, 2007.

14. Liu J, Qu R, Ogura M, Shibata T, Harada H and Hiraoka M: Real-time imaging of hypoxia-inducible factor-1 activity in tumor xenografts. J Radiat Res (Tokyo) 46: 93-102, 2005.

15. Ou G, Itasaka S, Zeng L, et al: Usefulness of HIF-1 imaging for determining optimal timing of combining bevacizumab and radiotherapy. Int J Radiat Oncol Biol Phys 75: 463-467, 2009.

16. Rapisarda A, Hollingshead M, Uranchimeg B, et al: Increased antitumor activity of bevacizumab in combination with hypoxia inducible factor-1 inhibition. Mol Cancer Ther 8: 1867-1877, 2009.

17. Rapisarda A, Zalek J, Hollingshead M, et al: Scheduledependent inhibition of hypoxia-inducible factor-1alpha protein accumulation, angiogenesis, and tumor growth by topotecan in U251-HRE glioblastoma xenografts. Cancer Res 64: 6845-6848, 2004.

18. Snoeks TJ, Lowik CW and Kaijzel EL: 'In vivo' optical approaches to angiogenesis imaging. Angiogenesis 13: 135-147, 2010

19. Yeom CJ, Chung JK, Kang JH, et al: Visualization of hypoxiainducible factor-1 transcriptional activation in C6 glioma using luciferase and sodium iodide symporter genes. J Nucl Med 49: 1489-1497, 2008

20. Saramaki OR, Savinainen KJ, Nupponen NN, Bratt O and Visakorpi T: Amplification of hypoxia-inducible factor 1alpha gene in prostate cancer. Cancer Genet Cytogenet 128: 31-34, 2001.
21. Ma $\mathbf{J}$ and Waxman DJ: Dominant effect of antiangiogenesis in combination therapy involving cyclophosphamide and axitinib. Clin Cancer Res 15: 578-588, 2009.

22. Harris AL: Hypoxia: a key regulatory factor in tumour growth. Nat Rev Cancer 2: 38-47, 2002.

23. Cuevas Y, Hernandez-Alcoceba R, Aragones J, et al: Specific oncolytic effect of a new hypoxia-inducible factor-dependent replicative adenovirus on von Hippel-Lindau-defective renal cell carcinomas. Cancer Res 63: 6877-6884, 2003.

24. Kizaka-Kondoh S, Itasaka S, Zeng L, et al: Selective killing of hypoxia-inducible factor-1-active cells improves survival in a mouse model of invasive and metastatic pancreatic cancer. Clin Cancer Res 15: 3433-3441, 2009.

25. Krohn KA, Link JM and Mason RP: Molecular imaging of hypoxia. J Nucl Med 49 (Suppl 2): S129-S148, 2008

26. Lehmann S, Stiehl DP, Honer M, et al: Longitudinal and multimodal in vivo imaging of tumor hypoxia and its downstream molecular events. Proc Natl Acad Sci USA 106: 14004-14009, 2009.

27. Raman V, Artemov D, Pathak AP, et al: Characterizing vascular parameters in hypoxic regions: a combined magnetic resonance and optical imaging study of a human prostate cancer model. Cancer Res 66: 9929-9936, 2006.

28. Kaelin WG Jr: The von Hippel-Lindau tumour suppressor protein: O2 sensing and cancer. Nat Rev Cancer 8: 865-873, 2008.

29. Kondo K, Kim WY, Lechpammer M and Kaelin WG Jr: Inhibition of HIF2alpha is sufficient to suppress pVHL-defective tumor growth. PLoS Biol 1: E83, 2003.

30. Stiehl DP, Wirthner R, Koditz J, Spielmann P, Camenisch G and Wenger RH: Increased prolyl 4-hydroxylase domain proteins compensate for decreased oxygen levels. Evidence for an autoregulatory oxygen-sensing system. J Biol Chem 281: 23482-23491, 2006.

31. Greenaway J, Henkin J, Lawler J, Moorehead R and Petrik J: ABT-510 induces tumor cell apoptosis and inhibits ovarian tumor growth in an orthotopic, syngeneic model of epithelial ovarian cancer. Mol Cancer Ther 8: 64-74, 2009.

32. Campbell NE, Greenaway J, Henkin J, Moorehead RA and Petrik J: The thrombospondin-1 mimetic ABT-510 increases the uptake and effectiveness of cisplatin and paclitaxel in a mouse model of epithelial ovarian cancer. Neoplasia 12: 275-283, 2010.

33. Harada H, Kizaka-Kondoh S and Hiraoka M: Optical imaging of tumor hypoxia and evaluation of efficacy of a hypoxia-targeting drug in living animals. Mol Imaging 4: 182-193, 2005.

34. Moroz E, Carlin S, Dyomina K, et al: Real-time imaging of HIF-1alpha stabilization and degradation. PLoS One 4: e5077, 2009. 